\title{
Video Article \\ Harvest of Endothelial Cells from the Balloon Tips of Swan-Ganz Catheters after Right Heart Catheterization
}

\author{
Michael J. Passineau ${ }^{1}$, Phillip H. Gallo ${ }^{1}$, Gretchen Williams ${ }^{1}$, Reina Perez ${ }^{1}$, Raymond L. Benza ${ }^{1}$ \\ ${ }^{1}$ Cardiovascular Institute, Allegheny Health Network
}

Correspondence to: Michael J. Passineau at Michael.Passineau@ahn.org

URL: https://www.jove.com/video/58353

DOI: doi:10.3791/58353

Keywords: Medicine, Issue 143, Right heart catheterization, pulmonary hypertension, Swan-Ganz catheter, endothelial cells, pulmonary endothelium, heart failure

Date Published: 1/23/2019

Citation: Passineau, M.J., Gallo, P.H., Williams, G., Perez, R., Benza, R.L. Harvest of Endothelial Cells from the Balloon Tips of Swan-Ganz Catheters after Right Heart Catheterization. J. Vis. Exp. (143), e58353, doi:10.3791/58353 (2019).

\section{Abstract}

A variety of pathologies lead to pulmonary hypertension $(\mathrm{PH})$, which is defined as a mean pulmonary artery pressure exceeding $25 \mathrm{mmHg}$ at rest. To further diagnose and manage $\mathrm{PH}$, patients undergo repeated right heart catheterizations (RHC) wherein a Swan-Ganz catheter is advanced into a branch of the pulmonary artery and a balloon is inflated to wedge the catheter tip. This article illustrates a protocol whereby pulmonary artery endothelial cells (PAECs) may be harvested from the balloon tips of Swan-Ganz catheters after RHC, and purified with an antiCD146 affinity column technique to purify putative PAECs. These cells might be used to provide an in situ snapshot of the biological state of the pulmonary vasculature endothelium to complement hemodynamic measurements obtained during RHC. Harvested and purified PAECs may be used for either cell culture or for subsequent analytical assays such as flow cytometery.

\section{Video Link}

The video component of this article can be found at https://www.jove.com/video/58353/

\section{Introduction}

Current U.S. and European guidelines require right heart catheterization for the diagnosis of pulmonary hypertension ${ }^{1}$. Physicians and scientists seeking to understand this disease desire biological samples from the pulmonary vasculature to complement the hemodynamic data that is gathered during RHC. Unfortunately, the risk associated with pulmonary vascular biopsy is very high, and therefore biological samples of the pulmonary vasculature are rarely if ever obtained from living patients undergoing RHC. While much insight has been gained from pulmonary vascular tissue obtained from cadaveric or explanted lungs, these samples are unable to reflect the biology of the $\mathrm{PH}$ disease process as it is unfolding in a living patient. In 2013, we first reported that pulmonary artery endothelial cells (PAECs) are denuded from the branches of the pulmonary artery by physical contact with the balloon tip of the Swan-Ganz catheter during RHC, and can be recovered in small numbers from the catheter tips after the procedure ${ }^{2}$.

This article illustrates our technique for the removal and purification of pulmonary artery endothelial cells (PAECs) from the balloon tips of SwanGanz catheters following right heart catheterization. Heretofore, the methodology for performing this harvest technique has been described in insufficient detail in the literature to ensure uniform results between laboratories performing the technique. This article is intended to overcome this deficiency, offering visualized demonstration of the technique, from bedside collection of catheter tips to the final product of purified CD146+ endothelial cells. This technique should aid in advancing research into the pathobiology of various etiologies of $\mathrm{PH}$, and possibly result in the development of new diagnostic assays to complement hemodynamic data gathered during RHC.

The importance of this technique to the field of pulmonary vascular medicine and research is that it provides access to a snapshot of the biology of the pulmonary endothelium in situ and in contrast to earlier studies on explanted or cadaveric lungs, does not represent an endpoint for the subject's lung. Recently, we have shown that an anti-apoptotic biomarker in these cells may have usefulness in more precisely phenotyping related varieties of pulmonary hypertension ${ }^{3}$. The yield of cells obtained is relatively small, $\sim 5,000-25,000$ PAECs per sample, and these cells are difficult (but not impossible) to culture. We believe that clever clinicians and researchers will find synergies between these companion techniques in order to advance pulmonary vascular research, diagnostics and prognostics.

\section{Protocol}

All studies described in this article were approved by the Institutional Review Board of the Allegheny Health Network. All patients provided informed consent before the administration of sedative agents and were de-identified by being assigned a study number. 


\section{Bedside Collection}

NOTE: Everything in this protocol should be done on ice, using pre-chilled solutions. It is important to induce cold stasis in the cells to preserve their in situ biological phenotype. This protocol involves the handling of blood and cells obtained from humans and therefore universal precautions should be observed.

1. After withdrawal of the catheter from the sheath, immediately immerse the catheter in a $50 \mathrm{~mL}$ centrifuge tube containing $40 \mathrm{~mL}$ of chilled sterile saline. Swirl the catheter several times to remove blood, and flush the line if necessary to remove blood retained within the line.

2. Cut off the distal $5 \mathrm{~cm}$ of the cath tip and allow it to fall into the $50 \mathrm{~mL}$ centrifuge tube containing chilled saline. Immediately remove the cath tip with forceps and transfer it to a $15 \mathrm{~mL}$ centrifuge tube containing $15 \mathrm{~mL}$ of endothelial cell (EC) growth medium.

3. Seal the tube, place it on ice and transfer to the lab to prepare for processing or shipping. It is critical that the balloon stays immersed in medium, so make sure there is minimal headspace in the $15 \mathrm{~mL}$ tube by filling it completely with EC growth medium.

4. Process the sample immediately by proceeding to Step 2.1 or seal and ship the sample on wet ice overnight for processing at a laboratory skilled in this protocol. If cells are ultimately going to be used for cell culture, it is strongly recommend that the cath tip be immediately processed at the same site at which it was collected.

\section{Cell Harvest}

NOTE: Everything in this protocol should be done on ice, using pre-chilled solutions. The cells are in cold stasis, and it is important to avoid awakening them until they are ready to be fixed or cultured. This entire protocol should be performed in a laminar flow hood, using sterile instruments, if the ultimate intent is culture of the harvested PAECs. If culture is not planned (e.g., flow cytometry analysis), it is not necessary to observe sterile precautions.

1. Add $5 \mathrm{~mL}$ of cell detachment solution to a $15 \mathrm{~mL}$ centrifuge tube; place on ice.

2. Draw $1 \mathrm{~mL}$ of $\mathrm{EC}$ media from that remaining in the bedside collection tube into the $1 \mathrm{~mL}$ syringe.

3. Quickly complete steps 2.3 through 2.5 . Grip the cut end of the catheter tip with hemostats.

4. Carefully insert the needle of the $1 \mathrm{~mL}$ syringe into the "white" hole opposite the guide wire ("black hole"). Take great care to avoid injury with the needle.

5. Transfer the cath tip into the $15 \mathrm{~mL}$ centrifuge tube containing the cell detachment solution.

6. Inflate the balloon, being careful not to burst it; inflating the balloon with approximately $200 \mu \mathrm{L}$ of media works well.

7. Allow the catheter to sit in cell detachment solution on ice for $5 \mathrm{~min}$ with the balloon inflated. If the balloon deflates on its own during the 5 min incubation, this is acceptable.

8. Pour the contents of the $15 \mathrm{~mL}$ centrifuge tube into the $100 \mathrm{~mm}$ dish. Place the balloon in the cell detachment solution "puddle".

9. Scrape the inflated balloon with a cell scraper. The cell scraper can be drawn around the balloon in a circular motion or a brushing motion. Focus on the "poles" of the balloon, where it meets the catheter. Ensure that the cell scraper contacts the entire surface of the balloon at least twice. Rinse the cell scraper in the cell detachment solution "puddle" periodically.

10. Deflate the balloon and scrape it with a brushing motion. Rinse the cell scraper in the cell detachment solution "puddle".

11. Discard the cath tip, needle and cell scraper in the appropriate waste container.

12. Pour the cell detachment solution containing the cell harvest into a $50 \mathrm{~mL}$ centrifuge tube. Shake the collection tube and pour the remaining media from the collection tube into the collected sample as well.

13. Centrifuge the sample at $650 \times g$ at $4{ }^{\circ} \mathrm{C}$ for $10 \mathrm{~min}$. It is important to note that oftentimes a pellet will NOT be seen. It can be helpful to mark the location where a pellet would be seen, if visible, to avoid aspirating the pellet in the event it is too small to be seen.

NOTE: Normally, a pellet is only seen when the sample has been contaminated by blood. Blood contamination is immaterial to the ultimate harvest product because blood fails to bind the CD146 beads and washes through the column. Extensive testing has demonstrated that the specific brand of $1.5 \mathrm{~mL}$ microcentrifuge tubes referenced in the Table of Materials minimizes cell loss relative to other tubes utilized during protocol optimization.

14. Carefully aspirate media, and avoid disturbing or aspirating the pellet.

\section{Cell Purification}

NOTE: Everything in this protocol should be done on ice, using pre-chilled solutions. The cells are in cold stasis, and it is important to avoid awakening them until they are ready to be fixed or cultured.

1. Resuspend the pellet in $1 \mathrm{~mL}$ of chilled red blood cell lysis solution, and transfer it to a $1.5 \mathrm{~mL}$ microfuge tube; gently rock for 10 min at $4{ }^{\circ} \mathrm{C}$.

2. Centrifuge for $5 \mathrm{~min}$ at $500 \times g$ and $4^{\circ} \mathrm{C}$. Aspirate the RBC lysis solution.

3. Resuspend the pellet in $60 \mu \mathrm{L}$ of PBS, $20 \mu \mathrm{L}$ of FCR blocker, and $20 \mu \mathrm{L}$ of anti-human CD146 microbeads. Incubate for $15 \mathrm{~min}$ at $4{ }^{\circ} \mathrm{C}$.

4. Add $1 \mathrm{~mL}$ of chilled PBS. Centrifuge for $5 \mathrm{~min}$ at $500 \times g$ and $4{ }^{\circ} \mathrm{C}$. Aspirate the supernatant and resuspend the pellet in $1 \mathrm{~mL}$ of chilled PBS.

5. Place a magnetic LS column on a magnetic rack (see Table of Materials for specific commercial identities of these items) at room temperature. Prime column by adding $1 \mathrm{~mL}$ of PBS and allow the entire volume to flow through. Discard the flow-through.

6. Place the sample in the magnetic column. Discard the flow-through.

7. Wash column with $3 \mathrm{~mL}$ of chilled PBS three times. Discard the flow-through from each wash.

8. Add $3 \mathrm{~mL}$ of chilled PBS to a $15 \mathrm{~mL}$ centrifuge tube. Remove the magnetic column from magnet, pour the $3 \mathrm{~mL}$ of PBS from the $15 \mathrm{~mL}$ centrifuge tube into the column and quickly place the column in the centrifuge tube to catch the flow-through. Immediately plunge with the plunger supplied with column. The sample is now purified, ready for fixation/staining, or culture. 


\section{Representative Results}

This protocol results in the purification of cells, from Swan-Ganz catheter tips after right heart catheterization, that bind to a CD146 selection column and have forward/side scatter profiles that are indistinguishable from cultured primary human pulmonary artery endothelial cells (Figure 1). Given that the catheter balloon is in physical contact with only the introducer sheath (a cell-free material), the pulmonary artery vessel wall, and the blood, data is provided herein to demonstrate that these cells are not derived from the blood (Figure 2), and therefore it is assumed that they are indeed derived from the pulmonary artery vessel wall. The reader should note that the anti-CD146 magnetic beads that are used to purify the cells remain adherent to the cell surface after the procedure, and thus it is suboptimal to attempt to analyze the cell harvests for CD146 as a marker of endothelial cells. CD31 or other endothelial markers would presumably be available for labeling and could be considered as alternatives.

Key elements of this protocol have been identified that may affect results. First, it is important to rinse the catheter tip at the bedside in order to remove blood. Figure 3 shows Side scatter (SSC)/Forward scatter (FSC) profiles of flow-through from the anti-CD146 column for one sample that was properly rinsed and one that was not. The column appears to exclude blood-derived cells from the harvests (Figure 2), but the rinsing

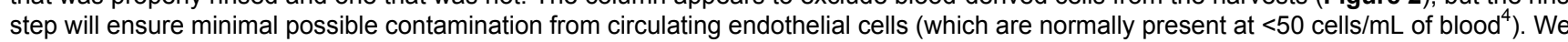
have observed that eluate from the CD146 column is completely clear, even in the case of very bloody catheter tip samples. This suggests that the column is able to handle significant amounts of blood without clogging or retaining non-CD146+ bloodborne cells.

Second, it is important to inflate the balloon tip at least once during the cell detachment solution/cell scraper step. Given that the putative PAECs on the balloon tip remain adherent despite the saline rinse step, it seems likely they may be partially trapped by folds in the vinyl balloon as it is deflated in preparation for removal from the patient. Presumably, the inflation step frees the PAECs, allowing them to be gently scraped away. It is not immediately clear one might test this hypothesis, but lower yields of cells have been observed when the balloon is not inflated (data not shown).

Finally, it is important to note that yields of PAECs derived from this method can vary considerably. Presumably, patient-, disease state- and physician-specific factors contribute to this variability, as the protocol does not contain steps that would be expected to result in major loss of source material. Table 1 shows representative CD146+ yields from a variety of PAEC harvests utilizing catheter tips obtained from a variety of patients with different disease states of pulmonary hypertension and a variety of physician operators.
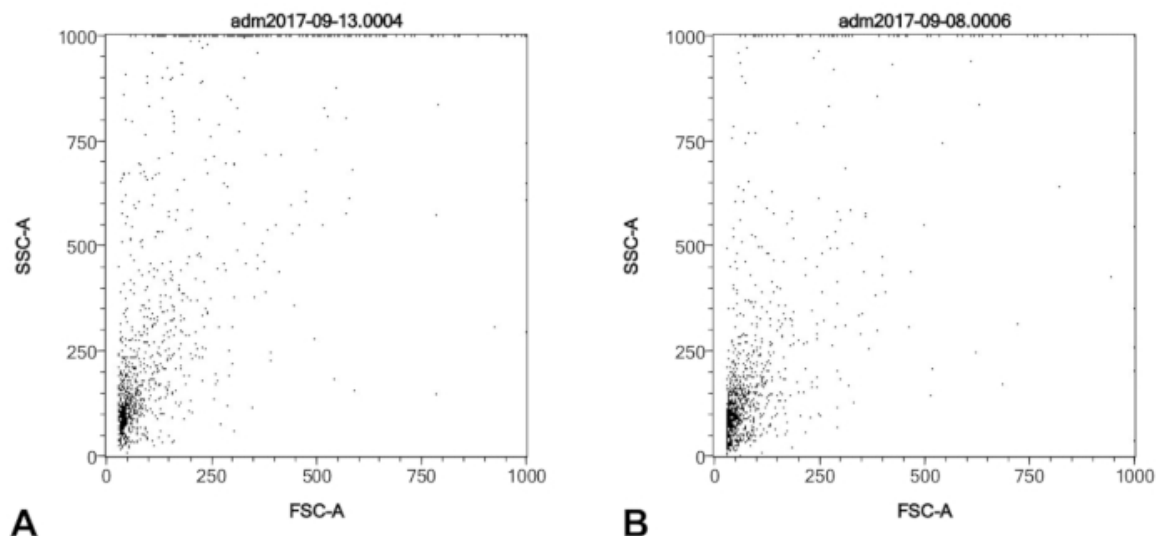

Figure 1: SSC/FSC profiles of a typical catheter balloon tip harvest from a pulmonary hypertension patient (A) and a similar number of cells from a commercially-acquired primary human PAEC culture (B). Both samples were purified with the anti-CD146 column, fixed, and permeablized as described in the protocol, prior to FACS analysis. Please click here to view a larger version of this figure. 

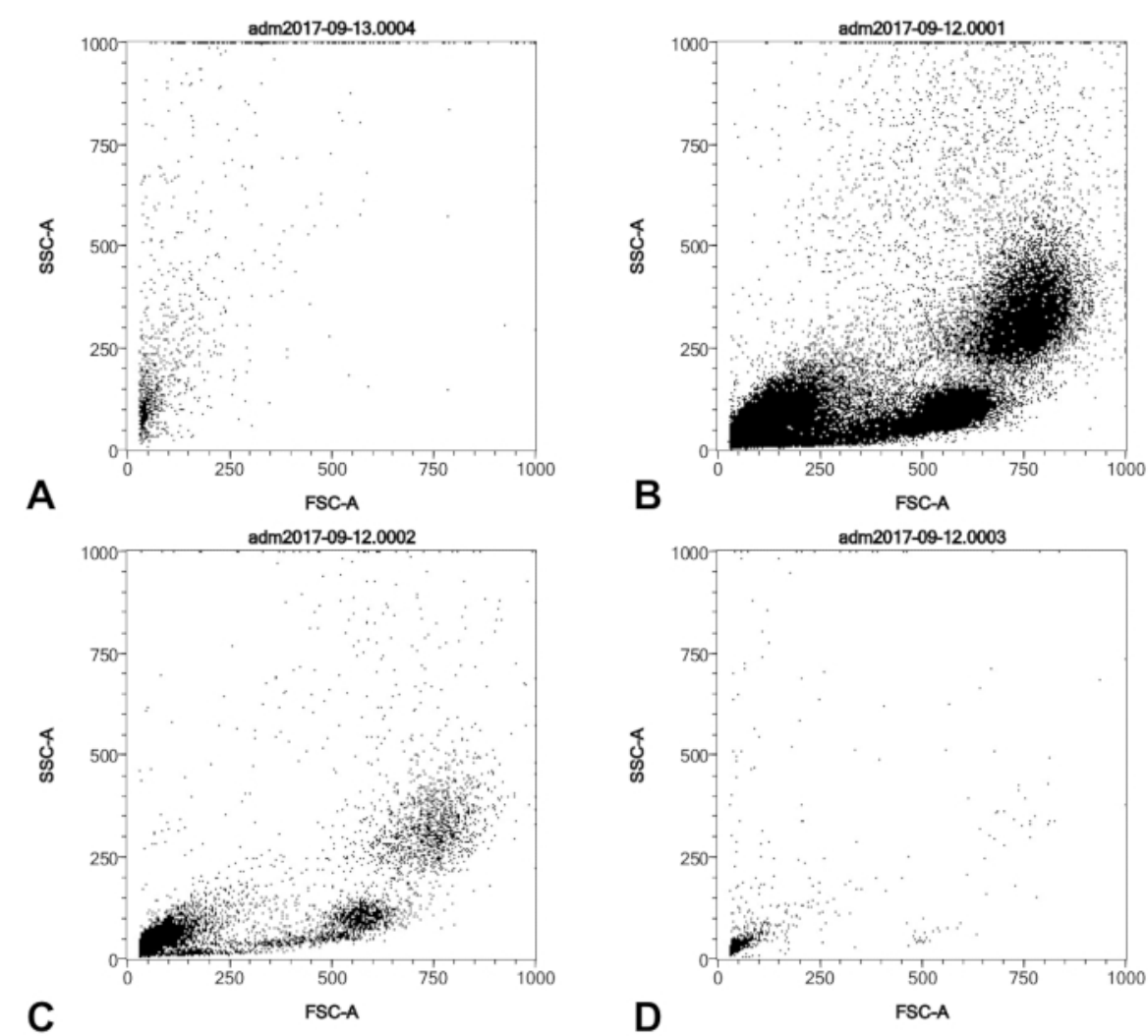

C

FSCA

D

Figure 2: SSC/FSC profiles of a typical catheter balloon tip harvest from a pulmonary hypertension patient (A) and serial 1:10 dilution of Ficoll gradient-purified human buffy coat (B, C, D). (A) was purified with the anti-CD146 column whereas (B-D) were not. All cell samples were fixed and permeablized as described in the protocol, prior to FACS analysis. Please click here to view a larger version of this figure. 

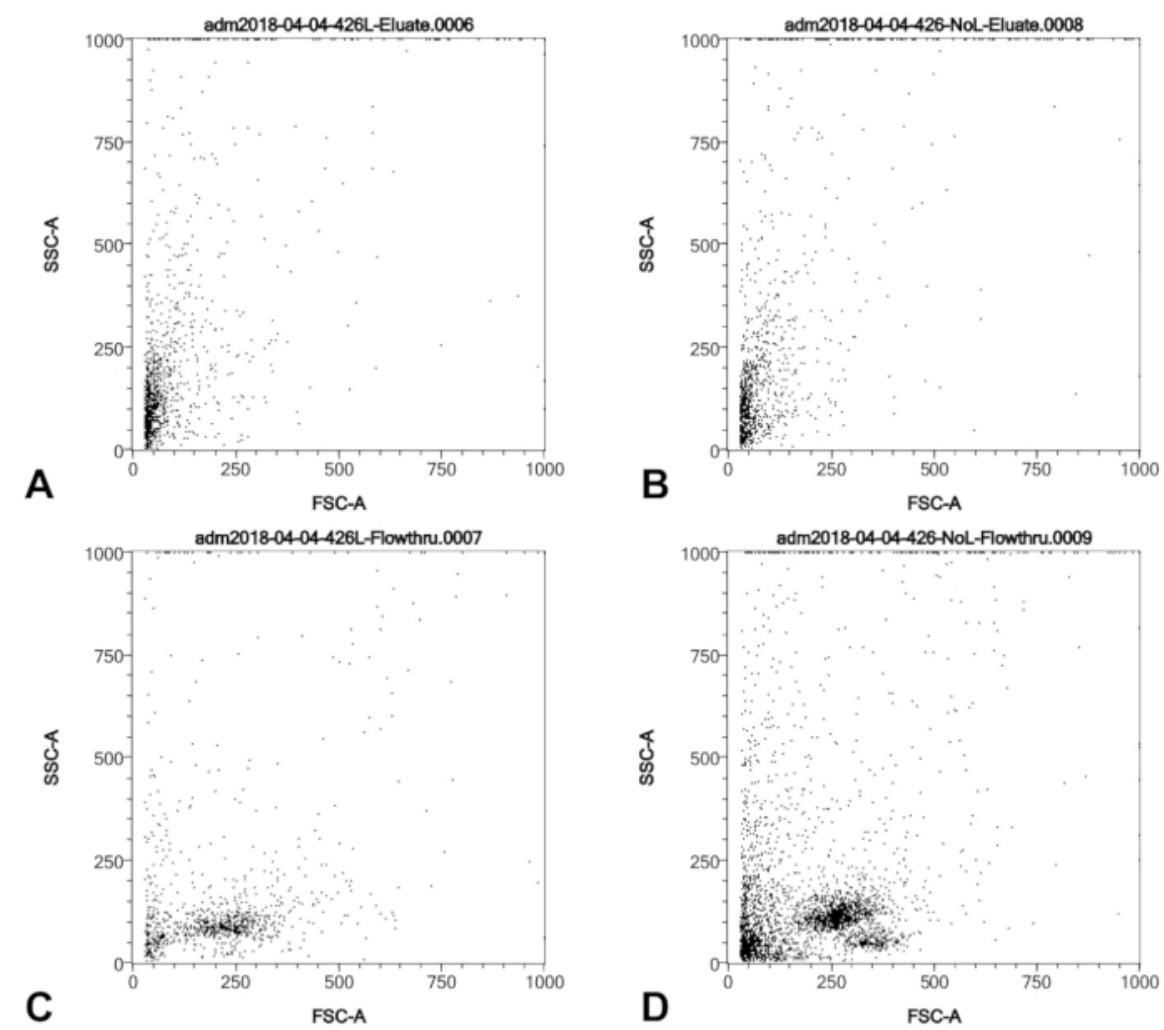

Figure 3: SSC/FSC profiles of a catheter balloon tip harvest from a pulmonary hypertension patient in which blood was visible on the catheter tip prior to processing. Prior to step 3.1 of the protocol, the sample was split and lysis buffer was added to one aliquot and withheld from the other. (A) and (C) show the SSC/FSC profiles of the lysed sample, both the cells retained by the column (A) and the flow-through from the column. (B) and (D) show the SSC/FSC profiles of the unlysed sample, both the cells retained by the column (B) and the flow-through from the column (D). We conclude that lysis removes some but not all of the leukocytes but that leukocytes are not retained by the column and are thus excluded from the final harvest, regardless of lysis. Please click here to view a larger version of this figure.

\begin{tabular}{|l|l|l|l|}
\hline Sample & Date & Cell Yield & Diagnosis \\
\hline 433 & $4 / 17 / 2018$ & 7364 & PH-Interstitial lung disease \\
\hline 279 & $4 / 17 / 2018$ & 7130 & PH-Scleroderma \\
\hline 432 & $4 / 13 / 2018$ & 3976 & PH-Multifactorial \\
\hline 431 & $4 / 13 / 2018$ & 3634 & HFrEF \\
\hline 390 & $4 / 13 / 2018$ & 5666 & PAH \\
\hline 423 & $3 / 23 / 2018$ & 9024 & HFrEF \\
\hline 272 & $3 / 23 / 2018$ & 7098 & HFrEF \\
\hline 408 & $2 / 27 / 2018$ & 4784 & HFrEF \\
\hline 403 & $2 / 14 / 2018$ & 10376 & PH-ASD+mitral disease \\
\hline 396 & $1 / 26 / 2018$ & 4890 & HFpEF-IPCPH \\
\hline 397 & $1 / 26 / 2018$ & 7672 & Normal hemodynamics \\
\hline 318 & $1 / 4 / 2018$ & 7002 & HFrEF-LVAD present \\
\hline
\end{tabular}

Table 1: CD146+ yields from a variety of PAEC harvests. Representative CD146+ yields from a variety of PAEC harvests utilizing catheter tips obtained from a variety of patients with different disease states of pulmonary hypertension and a variety of physician operators.

\section{Discussion}

The technique described in this article is best appreciated in the context of previous experimental paradigms for studying the pulmonary vascular endothelium. Previous attempts to obtain PAECs from humans have been limited to explanted/cadaveric lungs ${ }^{5}$ and a short-lived attempt to develop a catheter capable of pulmonary artery biopsy ${ }^{6}$. As noted in a recent review ${ }^{7}$, the present technique is distinguished from lung tissue harvest by its ability to obtain PAECs from a living human, and to do so at multiple time points in the progression of the disease and/or therapy. However, it should be noted that a companion technique has recently been reported whereby induced pluripotent stem cells (IPSCs) can be 
transdifferentiated into PAECs ${ }^{4,5}$. This technique produces abundant cultures of patient-specific PAECs and allows study of the effects of patientspecific genetic background on PAEC biology. Despite these advantages, these IPSC-derived PAECs cannot offer any information about the interaction between local microenvironment and patient-specific genetic background. Our own group has presented the view that the "cath tip" and IPSC techniques are likely to be synergistic in advancing the field ${ }^{8}$.

Within this present and historical context, the limitations of this technique should be noted. First, it is important to note that yields of PAECs derived from this method can vary considerably. Presumably, patient-, disease state- and physician-specific factors contribute to this variability, as the protocol does not contain steps that would be expected to result in major loss of source material. Table 1 shows representative CD146+ yields from a variety of PAEC harvests utilizing catheter tips obtained from a variety of patients with different disease states of pulmonary hypertension and a variety of physician operators. Second, cell yields are overall low, and their viability for culture has proven to be lower yet in our hands. While we have previously grown cultures of PAECs using this method ${ }^{1}$, we assume those results represent rescue of a subset of progenitor cells following die-off of most of the sample and is only intermittently successful in our hands. For example, it is not unusual to find only a few dozen adherent PAECs in culture the morning after a harvest has been plated. Often these micro-cultures do not survive to expansion. Further work is needed to optimize this protocol in a manner that will sustain the viability of PAECs and their conditions in culture to achieve the desired aim of propagation of patient-specific PAEC harvests. Finally, the CD146 positive selection we have adapted cannot exclude contamination with other CD146+ cells, such as circulating endothelial cells ${ }^{4}$, pericytes, smooth muscle cells and rare CD146+ T cells ${ }^{9,10}$. Nevertheless, these cells are presumed to be very few in number in samples collected in this manner, and CD146 is considerably more specific for this protocol than other cell markers of endothelial cells such as CD31 (which is also present on a variety of blood-borne cells including platelets ${ }^{11}$ ). Any future improvements aimed at improving specificity should be balanced against their potentially negative effect on yield.

\section{Disclosures}

MJP and RLB are inventors on patents claiming portions of the methods described.

\section{Acknowledgements}

The authors would like to thank the physicians and support staff of the Advanced Heart Failure and Pulmonary Hypertension service of Allegheny General Hospital for the provision of hundreds of catheter samples over several years that have facilitated the optimization of this methodology.

\section{References}

1. Galie, N. et al. 2015 ESC/ERS Guidelines for the diagnosis and treatment of pulmonary hypertension: The Joint Task Force for the Diagnosis and Treatment of Pulmonary Hypertension of the European Society of Cardiology (ESC) and the European Respiratory Society (ERS): Endorsed by: Association for European Paediatric and Congenital Cardiology (AEPC), International Society for Heart and Lung Transplantation (ISHLT). European Respiratory Journal. 46 (4), 903-975 (2015).

2. Pollett, J. B., Benza, R. L., Murali, S., Shields, K. J., \& Passineau, M. J. Harvest of pulmonary artery endothelial cells from patients undergoing right heart catheterization. Journal of Heart Lung Transplantation. 32 (7), 746-749 (2013).

3. Benza, R. L. et al. In situ expression of Bcl-2 in pulmonary artery endothelial cells associates with pulmonary arterial hypertension relative to heart failure with preserved ejection fraction. Pulmonary Circulation. 6 (4), 551-556 (2016).

4. Bull, T. M. et al. Circulating endothelial cells in pulmonary hypertension. Thrombosis and Haemostasis. 90 (4), 698-703 (2003).

5. Kaneko, F. T. et al. Biochemical reaction products of nitric oxide as quantitative markers of primary pulmonary hypertension. American Journal of Respiratory and Critical Care Medicine. 158 (3), 917-923 (1998).

6. Rothman, A. et al. Transvenous procurement of pulmonary artery smooth muscle and endothelial cells using a novel endoarterial biopsy catheter in a canine model. Journal of the American College of Cardiology. 27 (1), 218-224 (1996).

7. Savale, L., Guignabert, C., Weatherald, J., \& Humbert, M. Precision medicine and personalising therapy in pulmonary hypertension: seeing the light from the dawn of a new era. European Respiratory Reviews. 27 (148) (2018).

8. Kanwar, M., Raina, A., Passineau, M., \& Benza, R. Idiopathic Pulmonary Arterial Hypertension: Evolving Therapeutic Strategies. Seminars in Respiratory and Critical Care Medicine. 38 (5), 606-618 (2017).

9. Elshal, M. F., Khan, S. S., Takahashi, Y., Solomon, M. A., \& McCoy, J. P., Jr. CD146 (Mel-CAM), an adhesion marker of endothelial cells, is a novel marker of lymphocyte subset activation in normal peripheral blood. Blood. 106 (8), 2923-2924 (2005).

10. Elshal, M. F. et al. A unique population of effector memory lymphocytes identified by CD146 having a distinct immunophenotypic and genomic profile. BMC Immunolology. 829 (2007).

11. Newman, P. J. et al. PECAM-1 (CD31) cloning and relation to adhesion molecules of the immunoglobulin gene superfamily. Science. 247 (4947), 1219-1222 (1990). 